\title{
To evaluate the impact of initial chest radiograph on final outcome of ventilated patients
}

\author{
Indrajit Majumdar, Anil Sachdev, Dhiren Gupta, K. Chugh
}

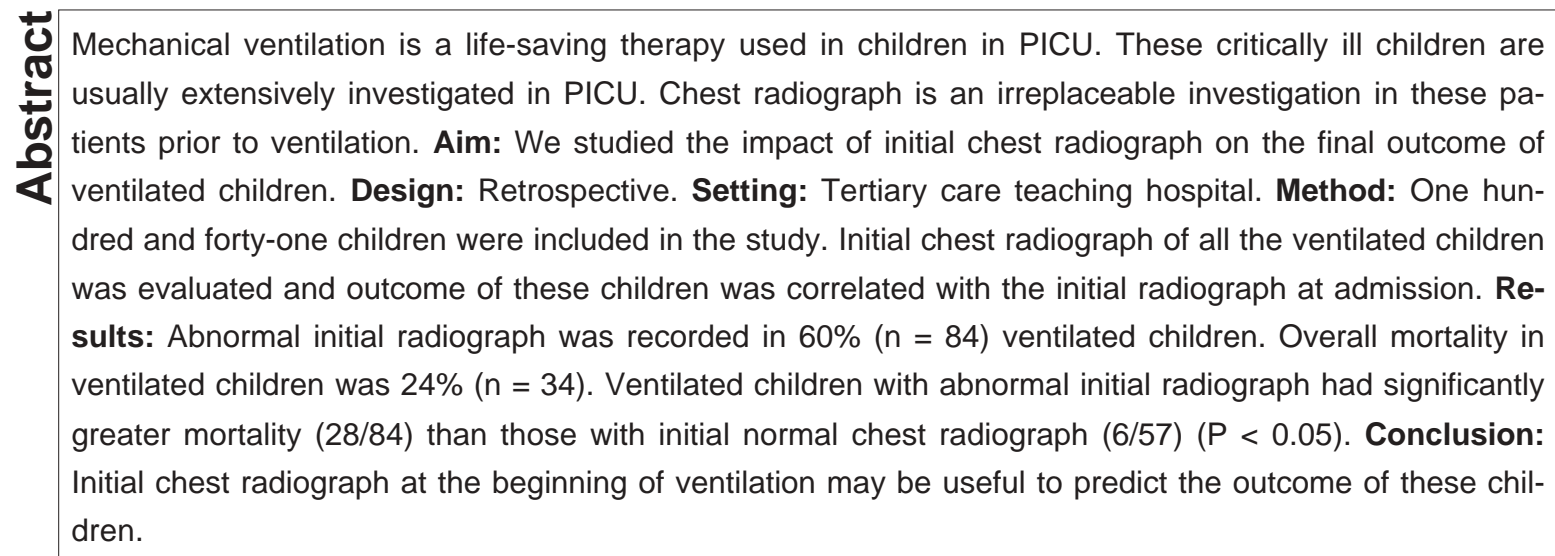

Key Words: Chest radiograph, Outcome, Mechanical ventilation, Pediatric intensive care unit

\section{Introduction}

Mechanical ventilation is common and essential therapy in pediatric intensive care unit (PICU). Children require ventilatory support for various conditions including respiratory and cardiovascular failure, shock with or without pulmonary involvement and depressed neurological status. Chest radiograph is routinely done in the mechanical ventilated patients. It remains one of the oldest noninvasive methods for identifying chest diseases. This tool is relatively inexpensive, low risk, and important for the intensivist to use in making an initial differential diagnosis. Timely intervention depends on the intensivists' ability to recognize the radiographic abnormalities commonly associated with acute pulmonary dis-

From:

Department of Pediatrics, Center for Child Health, Sir Ganga Ram Hospital, New Delhi, India

Correspondence:

Anil Sachdev,

Consultant Intensivist, Department of Pediatrics, Center for Child Health, Sir Ganga Ram Hospital, Old Rajinder Nagar, New Delhi - 110066, India.

E-mail: anilcriticare@hotmail.com ease. When linked with the history, presenting signs and symptoms, and a thorough physical assessment, the chest radiograph is key to appropriate diagnosis and management. ${ }^{[1]}$ Various scoring systems like pediatric risk of mortality (PRISM) score ${ }^{[2,3]}$ and prognostic indicators have been used to predict the outcome of PICU patients. However none of the commonly used scoring systems include the chest radiograph finding as a parameter in assessing the severity of illness or to predict the outcome of ventilated children despite being the most commonly used investigation in these children. The aim of this study was to evaluate if initial chest radiograph at admission or at the start of ventilation could be used to predict the outcome of ventilated children.

\section{Materials and methods}

This retrospective study was conducted at the PICU of a tertiary care multidisciplinary teaching hospital. Four hundred and fifty-three children were admitted in the 8bedded PICU between January 2000 and December 
2001. Of these, 141 children requiring ventilatory support for various conditions like respiratory failure, shock, and neurological abnormalities (Glasgow coma scale score less than 8 ) were included. Age of the children ranged from 3 days to 18 years with a median age of 10 months. Clinical data including primary diagnosis and laboratory parameters [Tables 1 and 2] along with the initial chest radiograph finding were recorded. The same panel of radiologists who regularly report all the pediatric radiographs had reported all the chest radiographs. Multi organ dysfunction syndrome (MODS) was diagnosed when two or more organs dysfunction was noted on the initial laboratory and radiological investigations. ${ }^{[4]}$

Table 1: Patient demography

Age (median age)

Male : Female

Primary diagnosis

Central nervous system

Meningo-Encephalitis

Head Injury

Infective polyneuritis

Status epilepticus

Respiratory system

Bronchopneumonia

Consolidation

Pleural effusion

Atelectasis

Gastrointestinal system

Fulminant hepatic failure

Intestinal obstruction

Cardiovascular system

Myocarditis

Congenital heart disease

Miscellaneous

Hemat-oncological

Dengue hemorrhagic feve

Diabetic ketoacidosis

Renal failure

Others

$\begin{array}{cc}\begin{array}{c}\text { Survivor } \\ (\boldsymbol{n}=107)\end{array} & \begin{array}{c}\text { Nonsurvivors } \\ (\boldsymbol{n}=34) \\ 10 \text { months }\end{array} \\ 2: 1 & 2: 1\end{array}$

$47(44 \%)$

17

10

12

8

$42(39 \%)$

24

10

5

$4(3.7 \%)$

$5(8.4 \%)$

4

1

$9(8.4 \%)$

2

1

1

3

2
$10(29.4 \%)$

4

5

0

$10(29.4 \%)$

1

1

$2(5.8 \%)$

0

$2(5.8 \%)$

1

1

$10(29.4 \%)$

6

1

0

3

0
Table 2: Laboratory parameters

\begin{tabular}{lccc}
\hline & $\begin{array}{c}\text { Survivors } \\
(\boldsymbol{n}=\mathbf{1 0 7})\end{array}$ & $\begin{array}{c}\text { Nonsurvivors } \\
(\boldsymbol{n}=\mathbf{3 4})\end{array}$ & $\boldsymbol{P}$ value \\
Hemoglobin $(\mathrm{g} / \mathrm{dl})$ & $9.3 \pm 2.15$ & $8.9 \pm 1.8$ & 0.33 \\
Blood urea nitrogen $(\mathrm{mg} / \mathrm{dl})$ & $22.5 \pm 10.3$ & $27 \pm 8.3$ & 0.02 \\
Serum creatinine $(\mathrm{mg} / \mathrm{dl})$ & $1.1 \pm 0.6$ & $1.2 \pm 0.8$ & 0.5 \\
Serum calcium $(\mathrm{mg} / \mathrm{dl})$ & $8.1 \pm 1.1$ & $7.9 \pm 1.3$ & 0.38 \\
Glucose $(\mathrm{mg} / \mathrm{dl})$ & $75.5 \pm 21.5$ & $85.2 \pm 30.2$ & 0.09 \\
$\mathrm{pH}$ & $7.26 \pm 0.8$ & $7.27 \pm 0.08$ & 0.5 \\
$\mathrm{PO}_{2}(\mathrm{~mm} \mathrm{Hg})$ & $78.6 \pm 51.1$ & $71.4 \pm 21.6$ & 0.25 \\
$\mathrm{PCO}_{2}(\mathrm{~mm} \mathrm{Hg})$ & $53.2 \pm 30.2$ & $51.2 \pm 23.2$ & 0.69 \\
Serum sodium $(\mathrm{mEq} / \mathrm{l})$ & $136.2 \pm 6.0$ & $139 \pm 5.5$ & 0.18 \\
Serum potassium $(\mathrm{meq} / \mathrm{l})$ & $4.6 \pm 0.9$ & $4.4 \pm 0.7$ & 0.18 \\
\hline
\end{tabular}

Data was analyzed using the Epi-info statistical package. Percentage was calculated for each variable. Chisquare test and student's $t$-tests were used, wherever applicable, for comparison. A $P$ value of $<0.05$ was considered significant.

\section{Results}

One hundred male and forty-one female ventilated children were included in the study. All the children had been directly admitted to the PICU. Age of the children ranged from 3 days to 18 years with a median age of 10 months. Infants less than 1 year of age constituted $52.6 \%$ of patients of which only one child was less than 1 month of age. The PICU mortality during the study period was $7.5 \%$. Overall mortality in the ventilated children was $24 \%(n=34)$.

The indications for ventilation were severe respiratory distress, respiratory failure, cardiovascular failure, shock with or without pulmonary involvement, and depressed neurological status with Glasgow coma scale score less than 8. Diseases of the central nervous system (CNS) $(40.4 \%)$ and respiratory system $(36.8 \%)$ accounted for majority of cases. Gastrointestinal (4.3\%) or cardiovascular disease $(4.9 \%)$ was the primary diagnosis in much fewer cases. Hemat-oncological diseases (5.7\%) included six cases of acute lymphoblastic leukemia and two cases of aplastic anemia. The six (4.2\%) cases of renal failure included three cases of hemolytic uremic syndrome and three cases of acute glomerulonephritis. Multiple organ systemic involvement was present in 56 children. One case of intestinal obstruction with shock was also included in the study.

None of the patients had required manual bag and mask ventilation for more than $\mathbf{3 0}$ min prior to ventilation. Endotracheal intubation was performed by an experienced personnel (intensivist, fellows or third year senior residents). Adequate premedication with midazolam for sedation and vecuronium for paralysis were used in all the patients. Lignocaine was additionally used for patients with suspected raised intracranial pressure. Siemens 900 or Siemens 300 ventilator was used for mechanical ventilation. All the patients were ventilated with volume controlled or pressure regulated volume-controlled ventilation modes except the neonate who received pressure-controlled ventilation. Synchronized intermittent mandatory ventilation (SIMV) with pres- 
sure support was used as a weaning mode once the indication of mechanical ventilation was reversed, patient was hemodynamically stable with no major electrolyte imbalance or obvious sepsis and was out of the effects of sedation and paralysis.

Laboratory parameters of both the survivors and nonsurvivors were comparable except the blood urea nitrogen [Table 2].

Initial chest radiograph was done in all 141 ventilated children within $2 \mathrm{~h}$ of admission into the PICU [Table 3]. Daily check radiographs were further done and the changes reported by the same panel of radiologists in all the patients during the period of ventilation.

Abnormal initial chest radiographs at the start of ventilation were seen in $60 \%$ of cases. Lobar/segmental collapse was the most common radiological abnormality $(40.4 \%)$ followed by bronchopneumonia (29.7\%). Combinations of abnormal radiographic findings were seen in 29 cases $(34.5 \%)$.

Of the 57 children with normal initial radiograph, 6 expired as compared to 28 among the 84 children with abnormal initial chest radiograph $(P<0.05)$.

Of the 34 children who expired, abnormal initial radiographs were seen in 28 (82\%) while, of the 107 children who survived abnormal initial radiographs were seen in only $56(52 \%)(P<0.01)$.

\section{Discussion}

The children who were admitted in PICU had multiple indications for ventilation, the most common being CNS disease (40\%). MODS was seen in $40 \%$ of the ventilated children. Thirty-seven percent of these children had respiratory pathology. However, chest radiographic abnormalities were seen in $60 \%$ of children with atelecta-

\begin{tabular}{lc}
\hline Table 3: Chest radiograph findings & \\
\hline Chest radiograph findings & Number of children \\
Abnormal & $84(60 \%)$ \\
Bronchopneumonia & $25(29.7 \%)$ \\
Lobar pneumonia & $18(21.4 \%)$ \\
Pleural effusion & $7(8.3 \%)$ \\
Collapse/atelectasis & $34(40.4 \%)$ \\
Combination pathology* & $29(34.5 \%)$ \\
Normal & $57(40 \%)$ \\
\hline${ }^{*}$ The numbers should not be included in the count as they are already \\
included in the primary pathology
\end{tabular}

sis/collapse accounting for majority of cases (34\%), closely followed by lobar and bronchopneumonia (33.7\%).

In the present study, the two groups of children (survivors and nonsurvivors) were comparable in median age, sex, hematological, and biochemical parameters.

Analysis of our data revealed that the children with abnormal initial radiograph had significantly higher mortality $(28 / 84)$ than those with normal initial radiograph $(6 / 57)(P<0.05)$.

Abnormalities in initial chest radiograph were also more common in ventilated children who had expired $(82 \%)$ than in those who had survived $(52 \%)(P<0.01)$ [Table 4]. The abnormal initial chest radiographic findings represent additional pathology in the ventilated children who might have been ventilated for non respiratory indications. This resulted in more difficult ventilation and higher ventilatory settings resulting in further ventilator induced injury. Subsequent prolongation of ventilation might have resulted in the development of new complications like ventilation-associated pneumonia, which would make weaning more difficult despite improvement in the initial pathology. This may account for the higher mortality in these children. However, in the absence of any similar study, this theory cannot be confirmed.

The number of patients in each primary diagnostic category is small as evident from [Table 1]. Analysis of correlation and effect of primary diagnosis and chest radiograph finding on outcome may not be fruitful. It is suggested that a prospective study may be conducted in a particular cohort with same primary illness.

Chest radiographs are routinely being done in all ventilated patients. The clinical value of routine chest radiographs in a PICU is well established. In a study by Hauser et al., physicians clinically failed to detect the chest findings and significant alterations in management would have been missed had the chest radiographs not been available. ${ }^{[5]}$ Another study by Quasney et al.$^{\left[{ }^{[6]}\right.}$ concluded

Table 4: Outcome of ventilated children with normal and abnormal initial chest radiograph

\begin{tabular}{lll}
\hline Ventilated children chest radiograph & $\begin{array}{l}\text { Survivors } \\
(n=107)\end{array}$ & $\begin{array}{l}\text { Nonsurvivors } \\
(n=34)\end{array}$ \\
Normal $(n=57)$ & $51(48 \%)$ & $6(18 \%)$ \\
Abnormal $(n=84)$ & $56(52 \%)$ & $28(82 \%)$
\end{tabular}


that routine chest radiographs are more useful in smaller, critically ill, ventilated children especially if there were one or more devices in situ and if active cardio-pulmonary problems were present.

Multiple scores are available to predict nosocomial pneumonia in $\mathrm{ICU}^{[7]}$ and nosocomial pneumonia in mechanically ventilated children ${ }^{[8]}$ However, predictors of outcome of ventilated children based on chest radiographs have not yet been studied. To the best of our knowledge, no study is available correlating the chest radiographic findings with variables presently being used in the outcome scoring systems like PRISM score which is the most commonly used scoring system to predict the outcome of PICU patients.

Univariate analysis of our data concludes that the chest radiograph may be an independent criterion to predict the outcome of ventilated children. However, in the absence of any similar studies, our observations could not be compared.

\section{Conclusion}

This study concludes that initial chest radiograph at beginning of ventilation not only provides additional information in ventilated children but may also be useful to predict their outcome. However, a larger study with multivariate analysis is required before this can be used as a predictor of outcome in mechanically ventilated children.

\section{References}

1. Marie A. Black, white and shades of gray: common abnormalities in chest radiographs. AACN Clin Adv Pract Ac Crit Care 2001;12:112-6.

2. Pollack MM, Ruttimann UE, Getson PR. Pediatric risk of mortality (PRISM) score. Crit Care Med 1988;16:1110-6.

3. Pollock MM, Patel KM, Ruttimann UE. PRISM III an updated pediatric risk of mortality score. Crit Care Med 1996;24:743-52.

4. Wilkinson JD, Pollack MM, Glass NL. Mortality associated with multiorgan failure and sepsis in ICU. J Pediatr 1987;111:324-8.

5. Hauser GJ, Pollack MM, Sivit CJ, Taylor GA, Bulas DI, Guion CJ. Routine chest radiographs in pediatric intensive care: a prospective study. Pediatrics 1989;83:465-70.

6. Quasney MW, Goodmann DM, Billow M, Chiu H, Easterling L, Frankel L, et al. Routine chest radiographs in pediatric intensive care units. Pediatrics 2001;107:241-8

7. Kropec A, Schulgen G. Scoring system for nosocomial pneumonia in ICUs. Intensive Care Medicine 1996;22:1155.

8. Drakulovic MB, Torres A. Supine body position as a risk factor for nosocomial pneumonias in mechanically ventilated children: A randomized trial. Lancet 1999;354:1851-8. 